\title{
Determinants of Diabetic Nephropathy among Diabetic Patients in General Public Hospitals of Tigray, Ethiopia, 2018/19
}

\author{
Teklewoini Mariye Zemicheal $\mathbb{D}^{1}{ }^{1}$ Degena Bahrey Tadesse, ${ }^{1}$ Hagos Tasew Atalay (D), \\ Girmay Teklay Weldesamuel, ${ }^{2}$ Gebrewahd Bezabh Gebremichael, ${ }^{3}$ Haben Nuguse Tesfay, ${ }^{4}$ \\ and Teklehaimanot Gereziher Haile ${ }^{5}$ \\ ${ }^{1}$ Department of Adult Health Nursing, School of Nursing, College of Health Science and Comprehensive Specialized Hospital, \\ Aksum University, Tigray, Ethiopia \\ ${ }^{2}$ Department of Pediatric Nursing, School of Nursing, College of Health Science and Comprehensive Specialized Hospital, \\ Aksum University, Tigray, Ethiopia \\ ${ }^{3}$ Department of Adult Health Nursing, School of Nursing, College of Health Science and Comprehensive Specialized Hospital, \\ Mekelle University, Mek'ele, Ethiopia \\ ${ }^{4}$ Department of Pediatrics, School of Medicine, College of Health Science and Comprehensive Specialized Hospital, \\ Aksum University, Tigray, Ethiopia \\ ${ }^{5}$ Department of Maternity and Child Health Nursing, School of Nursing, \\ College of Health Science and Comprehensive Specialized Hospital, Aksum University, Tigray, Ethiopia
}

Correspondence should be addressed to Teklewoini Mariye Zemicheal; teklewoini1@gmail.com

Received 3 March 2020; Revised 29 June 2020; Accepted 13 September 2020; Published 21 September 2020

Academic Editor: Franco Veglio

Copyright (c) 2020 Teklewoini Mariye Zemicheal et al. This is an open access article distributed under the Creative Commons Attribution License, which permits unrestricted use, distribution, and reproduction in any medium, provided the original work is properly cited.

\begin{abstract}
Background. Diabetic nephropathy is real damage resulting from having uncontrolled diabetes mellitus. Unmanaged diabetic nephropathy is one of the most leading causes of kidney failure. There is a scarcity of information on the determinants of diabetic nephropathy among diabetes mellitus patients in Ethiopia. Identification of the determinants can help devise a strategy to properly address the disease and its consequences. Therefore, this study was designed to assess the determinants of diabetic nephropathy among diabetes mellitus patients. Methods. Unmatched case-control study design with 168 cases and 672 controls with a mean age of 45.18 and 62.12, respectively, participated in the study. An interviewer-administered questionnaire was employed for data collection, and a systematic sampling technique was used to select the study participants. Data were entered into Epi data and exported to SPSS for data clarification and analysis. Binary logistic regression analysis was carried out to check the level of association between diabetic nephropathy and the independent variables. Results. Comorbidity (AOR: 4.96 at 95 CI: 1.77-13.87), hypertension (AOR: 6.33, 95\% CI: 2.51-16.02), poor glycemic control (AOR: 3.27, 95\% CI: 1.31, 8.21), age (AOR: 1.14, 95\%: 1.09-1.19), duration with diabetes mellitus since diagnosis (AOR: 1.83, 95 CI: 1.62-2.06), and nonadherence to diabetic medication (AOR: 3.3, 95\% CI: 1.34, 8.15), diet (AOR: 5.96, 95\%: 1.92-18.54), and exercise (AOR: 5.60, 95\% CI: 1.94-16.21) were the determinants of diabetic nephropathy. Conclusion. Adherence to medication, diet, and exercise should be empowered to achieve glycemic control and to prevent diabetic nephropathy. More attention has to be also given for old aged diabetic patients, long duration since diagnosis of diabetes mellitus, hypertension, and other comorbidities.
\end{abstract}

\section{Introduction}

Diabetic nephropathy (DN) is a clinical syndrome characterized by persistent microalbuminuria in concomitance with diabetes mellitus. It is diagnosed by the persistent increment of albumin or protein in urine when there is no other known renal disease $[1,2]$. It is a microvascular complication of diabetes mellitus characterized by persistent proteinuria, decreased glomerular filtration rate, and increased blood pressure [3].

Diabetic nephropathy typically develops after diabetes duration of 10 years, or at least 5 years with type 1 diabetes 
but may be present at the time of diagnosis of type 2 diabetes [4]. If early prevention methods are not applied, diabetic patients with microalbuminuria typically progress to proteinuria and overt diabetic nephropathy. Approximately $20-40 \%$ of diabetic patients develop microalbuminuria within 10-15 years of the diagnosis of diabetes, and about $80-90 \%$ of those with microalbuminuria progress to more advanced stages. After 15-20 years, macroalbuminuria occurs in approximately $20-40 \%$ of patients, and around half of them present with renal insufficiency within 5 years [5]. Strikingly, 40-45\% of patients with type 1 diabetes develop diabetic nephropathy and reach ESRD or die before its onset. Moreover, clinicians face a $30 \%$ prevalence of diabetic nephropathy among type 2 diabetic patients, with $45 \%$ of patients currently on dialysis having a primary diagnosis of diabetes [6].

The prevalence of diabetic nephropathy is still rising dramatically all over the world, with concomitant increases in associated mortality and cardiovascular complications [7]. Diabetic nephropathy is one of the most prevalent diabetes complications. With the global epidemic of diabetes mellitus, diabetic nephropathy has become an essential clinical and public health problem, with approximately one-third of them suffering from this long-term complication of diabetes [8]. It is a common and often devastating complication of diabetes mellitus associated with increased premature mortality and reduction in quality of life. It is a major factor in the development of chronic kidney disease and is the leading cause of end-stage renal disease [9] and the need for renal replacement therapy. It is also related to increased cardiovascular diseases and healthcare costs $[10,11]$.

The frequency of nephropathy in type 1 diabetes has a rather predictable prevalence (around 30-40\%), whereas in type 2 diabetes, it depends on several factors [12]. The main risks of diabetic nephropathy are hyperglycemia and arterial hypertension, but the genetic susceptibility in both type 1 and type 2 diabetes is of great importance. Glomerular hyperfiltration, smoking, dyslipidemia, proteinuria, and dietary factors are also responsible for the development of diabetic nephropathy [13].

Screening for diabetic nephropathy should be done at least once a year, by assessing urinary albumin and estimated glomerular filtration rate in patients with type 1 diabetes mellitus of $\geq 5$ years duration, in all type 2 diabetic patients, and in all diabetic patients with comorbid hypertension [4]. The initial treatment of diabetic nephropathy is prevention. Early detection of microalbuminuria and proper treatment may reverse or delay the progress of diabetic kidney disease. Patients should be treated to the lowest safe glucose level that can be obtained to prevent or control diabetic nephropathy. In addition to the aggressive treatment of elevated blood glucose, patients having diabetic nephropathy benefit from treatment with antihypertensive drugs [14].

$\mathrm{DN}$ was reported to be more common among diabetes patients in Africa than in the developed countries due to delayed diagnosis, insufficient screening and diagnostic services, poor control of blood sugar and other risk factors, and inadequate early stage care [6, 7]. Nonetheless, evidence supporting the burden of kidney disease in people with diabetes in Africa remains quite patchy and we are unaware of any attempts to synthesize existing data on the incidence of kidney disease in African diabetes populations.

Even though there are no clear definitions and there are different diagnostic criteria to assess the incidence and prevalence of diabetic nephropathy in Africa, it is a serious health threat for people with diabetes in the region with prevalence figures ranging from $11 \%$ to $83.7 \%$ [15]. The magnitude of diabetic complications in northern Africa ranges ranged from $6.7 \%$ to $46.3 \%$. The magnitude of diabetic nephropathy in sub-Saharan Africa among patients with type 2 diabetes ranges $10 \%$ to $49 \%$ [16]. The prevalence of diabetic nephropathy in Ethiopia ranges from $2 \%$ to $30 \%$ with an average of $15 \%$ [17].

In general, it has been found that despite preventive and therapeutic managements being available for diabetic nephropathy, a significant number of patients develop diabetic nephropathy. There is limited information on the burden of chronic kidney disease among diabetes mellitus patients in sub-Saharan Africa. Thus to prevent its devastating consequence, it is imperative to identify the determinants of diabetic nephropathy so that public health, preventive measures could be in place to decrease the morbidity and mortality of diabetic nephropathy. Hence, this study is aimed to assess the determinants of diabetic nephropathy among diabetic patients in general public hospitals of Tigray, Ethiopia.

\section{Methods}

2.1. Study Area and Period. Tigray is one of the nine regions of Ethiopia. The region has a total of 5,247,005 population with 2,660,002 females and 2,587,003 males [18]. The study was conducted in public hospitals of Tigray in which there are a total of fourteen general and two referral public hospitals. The study was conducted from September 1 to December 30, 2018, G.C.

2.2. Study Design. A hospital-based unmatched case-control study was conducted.

\subsection{Source Population}

Cases: patients with diabetic nephropathy in general public hospitals of Tigray at the time of data collection Controls: Diabetic patients without diabetic nephropathy in general public hospitals of Tigray at the time of data collection

\subsection{Study Population}

Cases: all the selected diabetic patients with diabetic nephropathy in the general public hospitals of Tigray

Controls: all the selected diabetic patients without diabetic nephropathy in the general public hospitals of Tigray 


\subsection{Eligibility Criteria}

\subsubsection{Inclusion Criteria}

Cases: all diabetic patients with diabetic nephropathy were included as cases

Controls: all diabetic patients without diabetic nephropathy were included as controls, respectively

2.5.2. Exclusion Criteria. Critically ill patients and pregnant mothers (gestational DM) were excluded from the study.

2.6. Sample Size Calculation. Epi Info software version 7.1.1 was used to calculate sample size considering the following parameters. $\quad$ Significance $=95 \%$; $\quad$ Power $=80 \%$; Odds ratio $=2.13$, which is the odds of diabetic nephropathy among diabetic patients with hypertension taken from a study conducted in Ayder Comprehensive Specialized Hospital, Tigray, Ethiopia, case to control ratio $=1: 4$, proportion of controls with exposure $=24.62 \%$, and proportion of cases with exposure $=41 \%$ [19]. The total sample size was 420 with 84 cases and 336 controls. Considering a design effect of 2 , the overall sample taken was 840 with 168 cases and 672 controls.

2.7. Sampling Technique and Procedure. Of the 14 general public hospitals in the regional state of Tigray, six hospitals were selected using the lottery method, and the calculated sample size was allocated proportionally to the selected hospitals based on the number of patients attending at the hospitals. The study subjects were selected using a systematic sampling technique every $K^{\text {th }}$ interval. $K^{\text {th }}$ interval was calculated by dividing the total number of cases and controls $(\mathrm{N})$ by the total sample size $(n)$ of cases and controls.

\subsection{Study Variables}

(i) Dependent variable

- Diabetic nephropathy

(ii) Independent variables

- Sociodemographic factors: sex, age, education status, residence, marital status, occupation, ethnicity, and religion; clinical characters: BMI status, duration with DM since diagnosis, hypertension, comorbidity, glycemic control, and behavioral factors (diabetic diet adherence, exercise adherence, medication adherence, alcohol intake, and smoking)

2.9. Data Collection Tool. A structured questionnaire was used for data collection. It had three parts. Part I: social demographic data; Part II: Respondents' health profile; Part III: Summary of Diabetic Care Activity (SDCA), which analyzes the self-practice domains of diabetes such as diet, exercise, smoking, and alcohol consumption. The reliability of the SDCA questionnaire was tested on similar studies conducted in Ethiopia [20, 21]. MMS (Modified Morse scale) was used to measure adherence to medication. The reliability of the MMS was tested among similar studies conducted in different regions of Ethiopia [22, 23]. The other variables such as weight, height, blood pressure, and fasting blood sugar were recorded using their standard measurements from the patients. Variables such as duration with diabetes, type of diabetes, presence of complications other than nephropathy, and fasting sugar level were taken from medical history records.

2.10. Data Collection Procedure. Data were collected by six trained nurses (BSc) and two supervisors (MSc). Cases and controls were identified from the records of diabetic patients by their identification number. Following the segregation of the cases and controls, data were extracted from each record review card and interviewing the study participants. Moreover, data related to weight, height, and blood pressure were obtained by measuring each of the study participants. Weight was measured in light clothing and without shoes in kilograms (kg) using a calibrated UNICEF Seca digital weighing scale and was checked every six patients by another calibrated UNICEF Seca digital weighing scale [24]. Height was measured using Stadiometer in centimeter $(\mathrm{cm})$ and measurement was checked every six patients by another Stadiometer. While measuring height, study subjects were kept in an erect position such that the back of the head, shoulder blades, buttocks, and heels making contact with the backboard of the Stadiometer [24]. Blood pressure was measured using a mercury sphygmomanometer with a cuff deflation rate of $2 \mathrm{mmHg}$. The average of two 5 minutes apart measurements of BP from the left arm in sitting position was recorded and each record was checked by another mercury sphygmomanometer [24].

2.11. Data Quality Control. A questionnaire prepared in English was translated to the local language (Tigrigna) by an individual who has good ability of the two-language translation. To ensure reliability, it was translated back to English by another individual fluent in both languages. A two-day training was given to data collectors and supervisors in Aksum town. A week prior to the actual data collection, the questionnaire was pretested on $5 \%$ of the total sample size in Suhul Hospital. The collected data were reviewed and checked for completeness and consistency by the supervisor and principal investigator on a daily bases during the data collection time.

2.12. Data Processing and Analysis. Data were entered into Epi data version 3.1 and analyzed using SPSS version 25 statistical software package. Descriptive statistics including proportion, percentage, ratios, frequency distribution, and mean and standard deviation were determined. Bivariate logistic regression analysis followed by multivariable logistic regression analysis was carried out to determine the association between the independent and outcome variables. Variables with $P$ value $\leq 0.2$ in the bivariate logistic regression analysis were entered into multivariable logistic 
regression. Statistical significance was declared using AOR with odds 95\% confidence interval (CI) at $P$ value $<0.05$.

\subsection{Operational Definitions}

Cases: patients diagnosed with diabetic nephropathy Controls: diabetic patients who had no diabetic nephropathy

Adherence to exercise: respondents who reported that they exercise for $>30$ minutes per day for at least five times per week

Adherence to the dietary regimen: this is the strict follow-up of the prescribed dietary regimen for $\geq 5$ days per week

Adherence to medication: this is the extent of adherence of drug taking behavior of a patient to the agreedupon recommendations by healthcare provider which was measured with Morisky's 8-item scale where an MMAS- 8 score of 6 and above was considered as adherent

Smoking-related adherence: respondents who reported to have never smoked

Good glycemic control: a glycemic control was considered to be good when a patient had $\mathrm{HbA} 1 \mathrm{c} \leq 7 \%$ and less than $8 \%$ for patients with comorbid vascular complications, age greater than 60, and a history of severe hypoglycemia [25]

Poor glycemic control: a glycemic control was considered to be poor when a patient had HbA1c greater than $7 \%$, and greater than $8 \%$ for those patients with comorbid vascular complications, age greater than 60 , a history of severe hypoglycemia [25]

\section{Results}

3.1. Sociodemographic Characteristics of the Respondents. There were a total of 840 participants (168 cases and 672 controls) with a response rate of $100 \%$. The mean age ( \pm standard deviation) of controls and cases was $45.18( \pm 8.35)$ and $62.12( \pm 13.45)$ with a minimum and maximum age of 23 and 80 years, respectively. Three hundred sixty-seven (54.6\%) controls and eighty-six (51.2\%) cases were male. Three hundred seventy-one (55.2\%) controls and eighty-three (66.8 $\%)$ cases were living in urban areas, and five hundred twentyone $(77.5 \%)$ of the controls and one hundred twenty-six (75\%) of the cases were married. One hundred fifty-one $(22.5 \%)$ controls and one hundred twenty-six (17.9\%) of the cases completed college and above. Six hundred fifty-nine (98.1\%) of the controls and all the cases $(100 \%)$ were Tegaru, and six hundred forty-six (96.1\%) of the controls and all of the cases were Orthodox Christian followers. Around one-fourth (25.75\%) of the total controls and forty-five $(28.7 \%)$ of the cases were private employees (See Table 1).

3.2. Health Profile of the Respondents. The mean duration of diabetes mellitus since diagnosis among cases and controls was $16.42( \pm 4.85)$ and $6.05( \pm 2.69)$, respectively. Five hundred nighty-nine $(89.1 \%)$ of the controls and one hundred twenty-nine $(76.8 \%)$ of the cases were members of EDA. Three-fourth (75\%) of the controls and one hundred forty-one $(83.9 \%)$ of the cases had type two DM. Three hundred sixty-one $(53.7 \%)$ of the controls and one hundred thirty-two $(78.6 \%)$ of the cases had a medically confirmed comorbidity, of which one hundred thirteen $(16.8 \%)$ of the controls and ninety-five (56.5\%) of the cases had hypertension. One hundred forty-one $(21 \%)$ of the controls and seventy $(41.7 \%)$ of the cases had a family history of diabetic mellitus. One-third of the controls (33.3\%) and seventy-two (42.9\%) of the cases were obese (see Table 2).

3.3. Behavioral Factors of the Respondents. Close to threefourth $(74.1 \%)$ of the controls and one hundred-two $(60.7 \%)$ of the cases were found adherent to their antidiabetic medications. But adherence to exercise was found in one hundred sixty-three $(24.3 \%)$ of the controls and fifty-three $(31.5 \%)$ of the cases. Two hundred fifty-one $(37.4 \%)$ of the controls and thirty-five $(20.8 \%)$ of the cases were adherent to the diabetic diet. More controls (48.2\%) than cases $(29.2 \%)$ had good glycemic control. Similarly, one hundred ninetyone $(28.4 \%)$ of the controls and fifty-nine $(35.1 \%)$ of the cases were alcohol consumers. Twenty-one $(3.1 \%)$ of the controls and five (3\%) of cases were smokers (see Table 3).

3.4. Determinants of Diabetic Nephropathy. In the bivariable logistic regression analysis, age, residence comorbidity, exercise, having hypertension, duration with diabetes since diagnosis, BMI status, diet, alcohol, family history of diabetic, adherence to medication, glycemic control, and membership of EDA had a $P$ value $<0.25$. However, in the multivariable analysis only, comorbidity [AOR: 4.96 at 95 CI: 1.77-13.87], having hypertension (AOR: 6.33 at 95\% CI: 2.51-16.02), poor glycemic control (AOR: 3.27 at 95\% CI: $1.31,8.21$ ), age (AOR: 1.14 at 95\%: 1.09-1.19), duration with DM since diagnosis (AOR: 1.83 at $95 \mathrm{CI}: 1.62-2.06$ ), and nonadherence to diabetic medication (AOR: 3.31 at $95 \% \mathrm{CI}$ : 1.34, 8.15), diet (AOR: 5.96 at 95\%: 1.92-18.54), and exercise (AOR: 5.60 at $95 \% \mathrm{CI}: 1.94-16.21$ ) were significant predictors of diabetic nephropathy (Table 4).

\section{Discussion}

As the age of diabetic patients increases one year, the chances of diabetic nephropathy increases by 1.14 times, which indicates that the odds of developing diabetic nephropathy increased by 3.74 times for ten years of diabetes age rise. A similar study conducted in Ayder Comprehensive Specialized Hospital, Tigray, showed that age was a determinant factor for diabetic nephropathy [19]. This relationship may be related to the diminished renal function on older DM patients compared to younger ones [26].

The odds of having diabetic nephropathy was 1.83 times higher, with every year increase in duration with diabetes. This indicates having diabetic nephropathy increased by 20.49 times more for five years increments of the duration with diabetes. This fact had been supported by different 
TABLE 1: Sociodemographic characteristics of the study participants on follow-up at public hospitals of Tigray, Ethiopia, 2019.

\begin{tabular}{|c|c|c|c|c|}
\hline Variables & Category & Controls $(n=672)$ & Cases $(n=168)$ & Total $(n=840)$ \\
\hline Age & Mean $( \pm S D)$ & $45.18(8.35)$ & $62.12( \pm 13.45)$ & \\
\hline \multirow{2}{*}{ Sex } & Male & $367(54.6 \%)$ & $86(51.2 \%)$ & $453(53.9 \%)$ \\
\hline & Female & $305(45.4 \%)$ & $82(48.8 \%)$ & $387(46.1 \%)$ \\
\hline \multirow{2}{*}{ Residence } & Urban & $371(55.2 \%)$ & $83(49.4 \%)$ & $454(54 \%)$ \\
\hline & Rural & $301(44.8 \%)$ & $85(50.6 \%)$ & $386(46 \%)$ \\
\hline \multirow{4}{*}{ Marital status } & Married & $521(77.5 \%)$ & $126(75 \%)$ & $647(77.0 \%)$ \\
\hline & Single & $43(6.4 \%)$ & $20(11.9 \%)$ & $63(7.5 \%)$ \\
\hline & Widowed & $48(7.1 \%)$ & $4(2.4 \%)$ & $52(6.2)$ \\
\hline & Divorced & $60(8.9 \%)$ & $18(10.7 \%)$ & $78(9.3 \%)$ \\
\hline \multirow{5}{*}{ Educational level } & Cannot read and write & $174(25.9 \%)$ & $45(26.8 \%)$ & $219(26.1 \%)$ \\
\hline & Can read and write & $89(13.2 \%)$ & $0(0.0 \%)$ & $89(10.6)$ \\
\hline & Primary y school & $144(21.4 \%)$ & $56(33.3 \%)$ & $200(23.8 \%)$ \\
\hline & Secondary school & $114(17.0 \%)$ & $37(22.0 \%)$ & $151(18.0 \%)$ \\
\hline & Colleague and above & $151(22.5 \%)$ & $30(17.9 \%)$ & $181(21.5 \%)$ \\
\hline \multirow{5}{*}{ Occupation } & House wife & $171(25.4 \%)$ & $32(19.0 \%)$ & $203(24.2 \%)$ \\
\hline & Governmental employee & $173(25.75)$ & $48(28.6 \%)$ & $221(26.3 \%)$ \\
\hline & Private employee & $193(28.7 \%)$ & $45(26.8 \%)$ & $238(28.3 \%)$ \\
\hline & Daily worker & $10(1.5 \%)$ & $2(1.2 \%)$ & $12(1.4 \%)$ \\
\hline & Farmer & $125(18.6 \%)$ & $41(24.4 \%)$ & $166(19.8 \%)$ \\
\hline \multirow{3}{*}{ Ethnicity } & Tigray & $659(98.1 \%)$ & $168(100.0 \%)$ & $827(98.5 \%)$ \\
\hline & Amara & $9(13 \%)$ & $0(0.0 \%)$ & $9(1.1 \%)$ \\
\hline & Oromo & $4(0.6 \%)$ & $0(0.0 \%)$ & $4(0.5 \%)$ \\
\hline \multirow{2}{*}{ Religion } & Orthodox & $646(96.1 \%)$ & $168(100.0 \%)$ & $814(96.9 \%)$ \\
\hline & Muslim & $26(3.9 \%)$ & $0(0.0 \%)$ & $26(3.1 \%)$ \\
\hline
\end{tabular}

TAвLE 2: Health profile of the study participants on follow-up at public hospitals of Tigray, Ethiopia, 2019.

\begin{tabular}{|c|c|c|c|c|}
\hline Variable & Category & Controls $(n=642)$ & Cases $(n=168)$ & Total $(n=840)$ \\
\hline Duration with DM & Mean $( \pm \mathrm{SD})$ & $6.05( \pm 2.69)$ & $16.42( \pm 4.85)$ & \\
\hline \multirow{2}{*}{ Membership EDA } & Yes & $599(89.1 \%)$ & $129(76.8 \%)$ & $728(86.7 \%)$ \\
\hline & No & $311(46.3 \%)$ & $36(21.4 \%)$ & $347(41.3 \%)$ \\
\hline \multirow{3}{*}{ Medication take } & Oral hypoglycemic & $527(78.4 \%)$ & $138(82.1 \%)$ & $665(79.2 \%)$ \\
\hline & Insulin & $37(5.5 \%)$ & $3(1.8 \%)$ & $40(4.8 \%)$ \\
\hline & Both & $108(16.1 \%)$ & $27(16.1 \%)$ & $135(16.1 \%)$ \\
\hline \multirow{2}{*}{ Family history } & Yes & $141(21.0 \%)$ & $70(41.7 \%)$ & $211(25.1 \%)$ \\
\hline & No & $531(79.0 \%)$ & $98(58.3 \%)$ & $629(74.9 \%)$ \\
\hline \multirow{2}{*}{ Type of DM } & Type one & $168(25 \%)$ & $27(16.1 \%)$ & $195(23.2 \%)$ \\
\hline & Type two & $504(75.0 \%)$ & $141(83.9 \%)$ & $645(76.8 \%)$ \\
\hline \multirow{2}{*}{ Comorbidity } & Yes & $361(53.7 \%)$ & $132(78.6 \%)$ & $493(58.7 \%)$ \\
\hline & No & $311(46.3 \%)$ & $36(21.4 \%)$ & $347(41.3 \%)$ \\
\hline \multirow{2}{*}{ Hypertension } & Yes & $113(16.8 \%)$ & $95(56.5 \%)$ & $208(24.8 \%)$ \\
\hline & No & $559(83.2 \%)$ & $73(43.5 \%)$ & $632(75.2 \%)$ \\
\hline \multirow{3}{*}{ BMI status } & Normal & $381(56.7 \%)$ & $77(45.8) \%$ & $458(54.5 \%)$ \\
\hline & Overweight & $67(10.0 \%)$ & $19(11.3 \%)$ & $86(10.2 \%)$ \\
\hline & Obese & $224(33.3 \%)$ & $72(42.9 \%)$ & $296(35.2 \%)$ \\
\hline
\end{tabular}

TABLe 3: Distribution of behavioral factors among study participants on follow-up at public hospitals of Tigray, Ethiopia 2019.

\begin{tabular}{|c|c|c|c|c|}
\hline Variables & Category & Controls $(n=672)$ & Cases $(n=168)$ & Total $(n=840)$ \\
\hline \multirow{2}{*}{ Adherence medication } & Adherent & $498(74.1 \%)$ & $102(60.7 \%)$ & $600(71.4 \%)$ \\
\hline & Nonadherent & $174(25.9 \%)$ & $66(39.3 \%)$ & $240(28.6 \%)$ \\
\hline \multirow{2}{*}{ Adherence diet } & Adherent & $251(37.4 \%)$ & $35(20.8 \%)$ & $286(34.0 \%)$ \\
\hline & Nonadherent & $421(62.6 \%)$ & $133(79.2 \%)$ & $554(66.0 \%)$ \\
\hline \multirow{2}{*}{ Adherence exercise } & Adherent & $163(24.3 \%)$ & $53(31.5 \%)$ & $216(25.7 \%)$ \\
\hline & Nonadherent & $509(75.7 \%)$ & $115(68.5 \%)$ & $624(74.3 \%)$ \\
\hline \multirow{2}{*}{ Smoker } & Yes & $21(3.1 \%)$ & $5(3.0 \%)$ & $26(3.1 \%)$ \\
\hline & No & $651(96.9 \%)$ & $163(97.0)$ & $814(96.9)$ \\
\hline \multirow{2}{*}{ Drinking alcohol } & Yes & $191(28.4 \%)$ & $59(35.1 \%)$ & $250(29.8 \%)$ \\
\hline & No & $481(71.6 \%)$ & $109(64.9 \%)$ & $590(70.2 \%)$ \\
\hline \multirow{2}{*}{ Glycemic control } & Good & $324(48.2 \%)$ & $49(29.2 \%)$ & 373 (44.4\%) \\
\hline & Poor & $348(51.8 \%)$ & $119(70.8 \%)$ & $467(55.6 \%)$ \\
\hline
\end{tabular}


TABle 4: Determinants of diabetic nephropathy among study participants on follow-up at public hospitals of Tigray region, Ethiopia, 2019.

\begin{tabular}{|c|c|c|c|c|c|}
\hline Variables & Category & Controls $(n=672)$ & Cases $(n=168)$ & COR $(95 \%$ CI $)$ & AOR $(95 \%$ CI $)$ \\
\hline Age & Mean $( \pm S D)$ & $45.18(8.35)$ & $62.12( \pm 13.45)$ & $1.19[1.16,1.23]$ & $1.14[1.09,1.19]^{*}$ \\
\hline Duration & Mean $( \pm S D)$ & $6.05( \pm 2.69)$ & $16.42( \pm 4.85)$ & $1.86[1.68,2.06]$ & $1.83[1.62,2.06]^{*}$ \\
\hline \multirow{2}{*}{ Comorbidity } & Yes & $361(53.7 \%)$ & $132(78.6 \%)$ & $3.14[2.12,4.71]$ & $4.96[1.77,13.87]^{*}$ \\
\hline & No & $311(46.3 \%)$ & $36(21.4 \%)$ & 1 & 1 \\
\hline \multirow{2}{*}{ Hypertension } & Yes & $113(16.8 \%)$ & $95(56.5 \%)$ & $6.44[4.46,9.28]$ & $6.33[2.51,16.02]^{*}$ \\
\hline & No & $559(83.2 \%)$ & $73(43.5 \%)$ & 1 & 1 \\
\hline \multirow{2}{*}{ Adherence medication } & Adherent & $498(74.1 \%)$ & $102(60.7 \%)$ & 1 & 1 \\
\hline & Nonadherent & $174(25.9 \%)$ & $66(39.3 \%)$ & $1.85[1.29,2.64]$ & $3.31[1.34,8.15]^{*}$ \\
\hline \multirow{2}{*}{ Adherence diet } & Adherent & $251(37.4 \%)$ & $35(20.8 \%)$ & 1 & 1 \\
\hline & Nonadherent & $421(62.6 \%)$ & $133(79.2 \%)$ & $2.26[1.51,3.39]$ & $5.96[1.92,18.54]^{*}$ \\
\hline \multirow{2}{*}{ Adherence to exercise } & Adherent & $163(24.3 \%)$ & $53(31.5 \%)$ & & 1 \\
\hline & Nonadherent & $509(75.7 \%)$ & $115(68.5 \%)$ & & $5.60[1.94,16.21]^{*}$ \\
\hline \multirow{2}{*}{ Glycemic control } & Good & $324(48.2 \%)$ & $49(29.2 \%)$ & 1 & 1 \\
\hline & Poor & $348(51.8 \%)$ & $119(70.8 \%)$ & $2.26[1.57,3.26]$ & $3.27[1.31,8.21]^{*}$ \\
\hline
\end{tabular}

*The determinants of diabetic nephropathy at $P$ value $<0.005$.

literature [27-32]. This finding could be explained by the fact that longer duration with DM may increase to have poor glycemic control and comorbidity, which affect renal functions through vascular damage.

The odds of diabetic nephropathy were 5.96 times higher among those nonadherent to diet compared to DM diet adherents. The finding of this study was congruent with a study conducted in Ayder Comprehensive Specialized Hospital, Tigray [33]. Adhering to dietary recommendations can enhance glycemic control and can reduce glycosylated hemoglobin (47) because DM-dietary foods have a low glycemic index which can reduce excess renal function pressure $(48,49)$.

The chances of diabetic nephropathy among respondents nonadherent to diabetic medications were 3.31 times higher compared to adherents to diabetic medications. This finding is consistent with a study done in Taiwan [34]. This may be due to the fact that nonadherence to diabetic medications contributes to poor glycemic control, which may intensify the gradual deterioration of renal functions, including renal disease in the final stages [35].

Poor glycemic control showed an association with the development of diabetic nephropathy. Similar significant associations were indicated in the findings of other previous studies done in South India, Oman, and westerns countries $[29,30,36,37]$. This is because poor glycemic control increases the glomerular filtration rate loss and albuminuria $[29,38]$. Besides, a high concentration of glucose in meningeal cells causes hypertrophy and increases gene expression and protein secretions which reduces the activity of metalloprotease enzyme which is responsible for the removal of west products [39].

Nonadherence to exercise was strongly significantly associated with diabetic nephropathy (AOR: 3.27 95\% CI $[1.31,8.21])$. A similar study result was found in Bangalore [32]. The reason may be due to the influences of DM-related exercise on several aspects of diabetic patients including blood glucose absorption, insulin action, and cardiovascular risk factors that preserve renal functions [40].
Having comorbidity also showed a strong significant association with diabetic nephropathy. Similar findings were found in other studies conducted in Bangalore and western countries [32, 37]. This may be due to the reason that comorbidities such as hypertension make arteries around the kidneys narrow and weaken and reduce the blood supply to the kidneys and stop removing waste products from the kidneys that may ultimately damage the kidney itself [41].

\section{Conclusion}

Older age, duration with DM since diagnosis, poor glycemic control, comorbidity, hypertension, and nonadherence to exercise, diet, and medication were found to be the predictors of diabetic nephropathy among DM patients. Adherence to diabetic management (medication, diet, and exercise) should be empowered to achieve glycemic control and to prevent diabetic nephropathy by strengthening information, education, and communication programs. More attention has to be given for older aged diabetic patients, patients with long duration since diagnosis of DM, hypertension, and other comorbidities. Another research should be carried out with another strong design to investigate the determinants of diabetic nephropathy broader social context and in a larger sample size.

\section{Abbreviations}

AOR: $\quad$ Adjusted odds ratio

COR: Crude odds ratio

CVD: Cardiovascular disease

DM: Diabetes Mellitus

FBS: $\quad$ Fasting blood sugar

HbA1c: Hemoglobin Alc

IEC: Information education and communication

IRB: Institutional review board

MMS: $\quad$ Modified Morse scale

SMBG: Self-monitoring of blood glucose

SDCA: Summary of diabetes care activity

UNICEF: United Nations International Child Fund. 


\section{Data Availability}

The data sets used and analyzed during the current study are available from the corresponding author upon request.

\section{Ethical Approval}

Ethical clearance was obtained from the Institutional Review Board of Aksum University, College of Health Science. Permission was obtained from the medical directors of the respective public hospitals to conduct the study.

\section{Consent}

Written consent was also obtained from each of the study participants. Respondents were allowed to refuse or discontinue participation at any time they want. Study participants were identified using codes and unauthorized persons were not having access to the collected data.

\section{Conflicts of Interest}

The authors declare no conflicts of interest regarding the publication of this paper.

\section{Authors' Contributions}

TM conceived and designed the study, analyzed the data, and wrote the manuscript. HT, GT, AG, GB, and HN took part in data analysis, drafting of the manuscript, and advising the whole research paper and also participated in the interpretation of the data and contributed to manuscript preparation. Similarly, all authors have read and approved the final version of the manuscript.

\section{Acknowledgments}

The authors would like to thank all study participants and data collectors for their contribution to the success of our study.

\section{References}

[1] W. Amogne, A. Reja, and A. Amare, "Diabetic foot disease in Ethiopian patients: a hospital based study," Ethiopian Journal of Health Development, vol. 25, no. 1, pp. 17-21, 2011.

[2] F. P. Schena and L. Gesualdo, "Pathogenetic mechanisms of diabetic nephropathy," Journal of the American Society of Nephrology, vol. 16, no. 3, pp. S30-S33, 2005.

[3] M. Abhilash and R. Augustine, Diabetes Mellitus and Human Health Care: A Holistic Approach to Diagnosis and Treatment, Taylor \& Francis, Oxfordshire, UK, 2014.

[4] P. H. Marathe, H. X. Gao, and K. L. Close, "American diabetes association standards of medical care in diabetes 2017," Journal of Diabetes, vol. 9, no. 4, pp. 320-324, 2017.

[5] C. Mora-Fernández, V. Domínguez-Pimentel, M. M. de Fuentes, J. L. Górriz, A. Martínez-Castelao, and J. F. Navarro-González, "Diabetic kidney disease: from physiology to therapeutics," The Journal of Physiology, vol. 592, no. 18, pp. 3997-4012, 2014.
[6] L. G. G. Gnudi and P. Ruggenenti, "The patient with diabetes mellitus," Oxford Textbook of Clinical Nephrology, Oxford University Press, Oxford, UK, 4th edition, 2016.

[7] M. Afkarian, M. C. Sachs, B. Kestenbaum et al., "Kidney disease and increased mortality risk in type 2 diabetes," Journal of the American Society of Nephrology, vol. 24, no. 2, pp. 302-308, 2013.

[8] A. T. Reutens and R. C. Atkins, "Epidemiology of diabetic nephropathy," Diabetes and the Kidney, vol. 170, pp. 1-7, Karger Publishers, Basel, Switzerland, 2011.

[9] K. Bennett and B. Aditya, "An overview of diabetic nephropathy: epidemiology, pathophysiology and treatment," Journal of Diabetes Nursing, vol. 19, no. 2, pp. 61-67, 2015.

[10] G. F. A. Assogba, C. Couchoud, C. Roudier et al., "Prevalence, screening and treatment of chronic kidney disease in people with type 2 diabetes in France: the ENTRED surveys (2001 and 2007)," Diabetes \& Metabolism, vol. 38, no. 6, pp. 558-566, 2012.

[11] G. L. Bakris, "Recognition, pathogenesis, and treatment of different stages of nephropathy in patients with type 2 diabetes mellitus," Mayo Clinic Proceedings, Elsevier, Amsterdam, Netherlands, 2011.

[12] R. C. Stanton, "Frontiers in diabetic kidney disease: introduction," American Journal of Kidney Diseases, vol. 63, no. 2, pp. S1-S2, 2014.

[13] S. John, "Complication in diabetic nephropathy," Diabetes \& Metabolic Syndrome: Clinical Research \& Reviews, vol. 10, no. 4, pp. 247-249, 2016.

[14] American Diabetes Association, "Microvascular complications and foot care: standards of medical care in diabetes," Diabetes Care, vol. 41, no. 1, pp. S105-S18, 2018.

[15] J. J. N. Noubiap, J. Naidoo, and A. P. Kengne, "Diabetic nephropathy in Africa: a systematic review," World Journal of Diabetes, vol. 6, no. 5, p. 759, 2015.

[16] D. Ganu, N. Fletcher, and N. Caleb, "Physical disability and functional impairment resulting from type 2 diabetes in subSaharan Africa: a systematic review," African Journal of Diabetes Medicine, vol. 24, no. 1, 2016.

[17] T. Nigatu, "Epidemiology, complications and management of diabetes in Ethiopia: a systematic review," Journal of Diabetes, vol. 4, no. 2, pp. 174-180, 2012.

[18] Wikipedia.org/wiki/, Regional Hospitals and Healthcare Facilities in Ethiopia 2013, https://en.wikipedia.org/wiki/List_ of_hospitals_in_Ethiopia.

[19] S. Hintsa, L. Dube, M. Abay, T. Angesom, and A. Workicho, "Determinants of diabetic nephropathy in ayder referral hospital, northern Ethiopia: a case-control study," PloS One, vol. 12, no. 4, 2017.

[20] K. K. Berhe, A. Demissie, A. B. Kahsay, and H. B. Gebru, "Diabetes self care practices and associated factors among type 2 diabetic patients in Tikur Anbessa specialized hospital, Addis Ababa, Ethiopia-a cross sectional study," International Journal of Pharmaceutical Sciences and Research, vol. 3, no. 11, 2012.

[21] K. K. Berhe, H. B. Gebru, H. B. Kahsay, and A. A. Kahsay, "Assessment of self care management and its associated factors among type 2 diabetes patients in mekelle hospital and ayder referral hospitals, mekelle city, Tigray, northern Ethiopia, 2012/13," Global Journal of Medical Research, vol. 17, 2017.

[22] A. Kassahun, F. Gashe, E. Mulisa, and W. A. Rike, "Nonadherence and factors affecting adherence of diabetic patients to anti-diabetic medication in Assela General Hospital, 
Oromia Region, Ethiopia," Journal of Pharmacy \& Bioallied Sciences, vol. 8, no. 2, 2016.

[23] S. M. Abebe, Y. Berhane, and A. Worku, "Barriers to diabetes medication adherence in North West Ethiopia," Springerplus, vol. 3, no. 1, p. 195, 2014.

[24] Centers for Disease Control and Prevention, National Health and Nutrition Examination Survey (NHANES) Anthropometry Procedures Manual, Createspace, Scotts Valley, CA, USA, 2014.

[25] A. Y. Y. Cheng and Canadian Diabetes Association Clinical Practice Guidelines Expert Committee, "Introduction," $\mathrm{Ca}$ nadian Journal of Diabetes, vol. 37, p. S361, 2013.

[26] R. D. Lindeman, J. Tobin, and N. W. Shock, "Longitudinal studies on the rate of decline in renal function with age," Journal of the American Geriatrics Society, vol. 33, no. 4, pp. 278-285, 1985.

[27] N. H. Cho, J. E. Shaw, S. Karuranga et al., "IDF Diabetes Atlas: global estimates of diabetes prevalence for 2017 and projections for 2045," Diabetes Research and Clinical Practice, vol. 138, pp. 271-281, 2018.

[28] V. Vimalkumar, C. A. Moses, and S. Padmanaban, "Prevalence \& risk factors of nephropathy in type 2 diabetic patients," International Journal of Collaborative Research on Internal Medicine \& Public Health, vol. 3, no. 8, 2011.

[29] R. Unnikrishnan, M. Rema, R. Pradeepa et al., "Prevalence and risk factors of diabetic nephropathy in an urban South Indian population: the Chennai Urban Rural Epidemiology Study (CURES 45)," Diabetes Care, vol. 30, no. 8, pp. 2019-2024, 2007.

[30] A. H. Alrawahi, S. G. A. Rizvi, D. Al-Riyami, and Z. Al-Anqoodi, "Prevalence and risk factors of diabetic nephropathy in omani type 2 diabetics in Al-dakhiliyah region," Oman Medical Journal, vol. 27, no. 3, p. 212, 2012.

[31] P. Prashanth, K. J. Sulaiman, G. Kadaha et al., "Prevalence and risk factors for albuminuria among type 2 diabetes mellitus patients: a Middle-East perspective," Diabetes Research and Clinical Practice, vol. 88, no. 3, pp. e24-e27, 2010.

[32] M. Ahmed, G. Kishore, H. Khader, and M. Kasturirangan, "Risk factors and management of diabetic nephropathy," Saudi Journal of Kidney Diseases and Transplantation, vol. 24, no. 6, p. 1242, 2013.

[33] M. T. Angamo, B. H. Melese, and W. Y. Ayen, "Determinants of glycemic control among insulin treated diabetic patients in Southwest Ethiopia: hospital based cross sectional study," PloS One, vol. 8, no. 4, Article ID e61759, 2013.

[34] P.-Y. Chang, L.-N. Chien, Y.-F. Lin, H.-Y. Chiou, and W.-T. Chiu, "Nonadherence of oral antihyperglycemic medication will increase risk of end-stage renal disease," Medicine, vol. 94, no. 47, 2015.

[35] T. Dunning, "Assessment and management of patients with diabetes mellitus," Smeltzer \& Bare's Textbook of MedicalSurgical Nursing, pp. 1219-1270, Wolters Kluwer Health, Philadelphia, PA, USA, 2010.

[36] R. Jayakumar, Risk Factors in Diabetic Nephropathy, Springer, Berlin, Germany, 2012.

[37] B. Satirapoj and S. G. Adler, "Prevalence and management of diabetic nephropathy in western countries," Kidney Diseases, vol. 1, no. 1, pp. 61-70, 2015.

[38] R. J. MacIsaac, G. Jerums, and E. I. Ekinci, "Effects of glycaemic management on diabetic kidney disease," World Journal of Diabetes, vol. 8, no. 5, p. 172, 2017.

[39] R. E. Gilbert and M. E. Cooper, "The tubulointerstitium in progressive diabetic kidney disease: more than an aftermath of glomerular injury?" Kidney International, vol. 56, no. 5, pp. 1627-1637, 1999.

[40] S. R. Chipkin, S. A. Klugh, and L. Chasan-Taber, "Exercise and diabetes," Cardiology Clinics, vol. 19, no. 3, pp. 489-505, 2001.

[41] A. J. M. Boulton, "What you can't feel can hurt you," Journal of Vascular Surgery, vol. 52, no. 3, pp. 28S-30S, 2010. 